Journal of Medical Genetics, 1983, 20, 316-318

\section{Familial Inherited Abnormalities}

Clinics in Gastroenterology, Volume 11, No 1. Edited by $\mathbf{J}$ T Harries. (Pp viii + 234; figures + tables. £10-75.) London, Philadelphia, Toronto: Saunders. 1982.

The title of this volume in the series of Clinics in Gastroenterology is rather misleading. It covers predominantly the biochemical aspects of genetically determined metabolic errors affecting the gastrointestinal tract, while the extent to which clinical aspects are discussed varies considerably. Comment on the genetics is often an unsupported statement of autosomal inheritance, though the general principles have already been covered in an excellent introductory chapter. The section on multifactorial inheritance should be particularly useful for gastroenterologists in view of the familial incidence of so many diseases of the gut. However, only 11 pages are devoted to these mainly more common disorders, including infantile pyloric stenosis, duodenal ulcer, coeliac disease, and inflammatory bowel disease. There is a useful short section on generalised syndromes in which the gut may be involved, such as pseudoxanthoma elasticum.

The major portion of the book deals with disorders of carbohydrate, electrolyte, nitrogen, vitamin, mineral, and lipid absorption. Considerable detail is given on the physiology and pathology of the absorptive process with extensive references. I found the chapter on mineral absorption particularly informative. Two of the longest chapters are on the pancreas and liver. They both show a better balance with rather less on the metabolic pathways, though details are included where known, but there is also some overlap, for example, cystic fibrosis and $\alpha 1$-antitrypsin deficiency. The chapter on exocrine diseases of the pancreas concentrates on cystic fibrosis, Schwachman's syndrome, and hereditary pancreatitis. Schwachman's syndrome, comprising exocrine pancreatic insufficiency, varying pancytopenia, and normal sweat electrolytes (which distinguishes it from cystic fibrosis) is the second most frequently recognised cause of pancreatic insufficiency in children. Analysis of sibships, segregation ratios, and familial incidence are stated to support an autosomal recessive mode of inheritance, though the factors leading to multisystem disease are unidentified. The chapter on the liver is very comprehensive with clinical descriptions as well as diagrams and tables, indicating the sites of metabolic blocks and $\frac{\Phi}{\Phi}$ the enzyme deficiency, for example, for the glycogen storage diseases.

Overall, there is an unevenness in content between $\vec{\circ}$ the different chapters. For example, the nature of the $\overrightarrow{\vec{\omega}}$ familial occurrence of coeliac disease is only $\stackrel{\sigma}{\omega}$ mentioned briefly, whereas abetalipoproteinaemia $\overline{\overline{3}}$ with the possible value of vitamin $E$ therapy is 0 dealt with at some length. This can only partly be $\stackrel{0}{0}$ accounted for by the rarity of some syndromes and the way in which 'nature's experiment' has presented. $\frac{\omega}{\sigma}$ However, as a source of information and references on the biochemical aspects of inherited disorders of $\supset$ absorption it is excellent. Its readersh/p is probably $\vec{\longrightarrow}$ limited and a more accurate title, such as inherited c. disorders of gastrointestinal metabolism, would $\frac{0}{\infty}$ have indicated more properly its scope.

A W Johnston $\vec{e}$

Histocompatibility Antigens. Structure and Function Receptors and Recognition Series B, Volume 14. S Edited by P Parham and J Strominger. (Pp xi +246 ; figures + tables. £27.50.) London, New York: Chapman and Hall. 1982.

Jan Klein ends this book with the following most illuminating analogy. If immunoglobulins are like arrows shot at very specific target antigens, the major histocompatibility complex (MHC) is almost certainly the archer who decides who is enemy and who is friend. Thus, antigens coded by genes in $:$ MHC are responsible for the 'restriction' of cell-cell 3 . interactions so as to produce specific immunological responses to foreign antigens while avoiding damage $₹$ to self antigens. No one is sure exactly how this 0 works, but it is certain that the presence of self antigen is necessary for the generation of clones of $\frac{}{2}$ $T$ cells that 'help' the development of an immune response and other clones that destroy antigenically or specific targets. The remarkable polymorphism of $N$ MHC antigens has, according to Klein, evolved N independently of speciation and this complexity is $\omega$ seemingly essential for vertebrate survival.

This volume aims to provide an up to date assess- $-\stackrel{\mathcal{O}}{\simeq}$ ment of knowledge of MHC antigens and their role in the immune system. It is not designed to be $a_{7}^{+}$ standard text book of the serology of genetics of ${ }^{\circ}$ MHC. It is aimed rather at the graduate level or $\overrightarrow{\mathbb{D}}$ higher and although never more complex or arcane $\underset{\mathbb{D}}{\stackrel{O}{O}}$ than the subject matter dictates, opportunities are $\varrho$ 